\title{
Síndrome de Peutz-Jeghers
}

\author{
Dr. Sejeio Ceresa $0 .^{1}$; Dr. Rafacl Salinas $G^{2}{ }^{2}$ Dr, Albcrto Salamon $\mathbf{I}^{2}$
}

\section{Peutz-Jeghers Syndrome}

Three patients with Peutz-Jeghers syndrome are described. Two of them presented with intestinal intussuseption. In the third casc the diagnosis was suspected by the melanic mucocutaneous manifestations. All had polypoid lesions of the gastrointestinal tract at $X$ Ray examination with barium cnemas. Fiber optic gastroduodenoscopy and colonoscopy showed the characteristic polypoid lesions. lindoscopic polypectomy was performed and its uscfulncss in stressed.

(Key words: Peutz-Jegher's Syndrome. Intussuseption, lntestinal poly posis).

El Sindrome de Peut $z$-Jeghers se caracteriza por la presencia de poliposis hamartomatosa del tracto gastrointestinal y pigmentación melánica mucocutánea. Se transmite por herencia autosómica dominante ${ }^{1,2}$. Los póljpos pueden encontrarse en toda la longitud del tracto gastrointestinal desde el esfínter gastroesofágico. Frecuentemente se les ubica en intestino delgador, 3 .

Se han descrito más de 300 casos de PeutzJeghers ${ }^{4}$. El diagnóstico se realiza antes de los 15 años en un tercio de estos $\operatorname{casos}^{4}$.

Entre 1982 y 1985 han corisultado tres pacientes con este sindrome en el Hospital Paula Jaraquemada cuya descripción constituye el objetivo de este trabajo.

\section{Casos Clínicos}

R.A.J., escolar de 10 años, ingresó al Servicio de Urgencia con dolor cólico abdominal intermitente, de veinticuatro horas de evolución acompañados de vómitos frecuentes. Tenia antecedentes de rectorragia desde los tres años de edad. En el examen físico mostraba palidez, manchas pigmentarias melánicas en los labios, boca, encias y paladar, palma de las manos, pulpejos y planta de los pies. En el abdomen se palpaban masas abdominales en la región para. mediana derecha y periumbilical y se observaban movimientos peristálticos intestinales. El tacto rectal no demostró sangramiento visible. Ingresó con los diagnósticos de síndrome de PeutzJeghers e invaginación intestinal. Durante la

1. Médico Departamento Gastroenterología Infantil. Hospital Paula Jaraquemada.

2. Médico Servicio Cirugía Infantil. Hospital Paula Jaraquemada. intervención quirúrgica se vio un asa intestinal con coloración anormal. aspecto necrótico y compromiso vascular, yue correspondía al ycyu no invaginado, cuya reducción resultó imposible: se realizó resección de más o menos $50 \mathrm{~cm}$. a una distancia de $25 \mathrm{~cm}$. del ángulo de Treitz y anastomosis término terminal. Se palparon otros pólipos en zonas de intestino normal que no fueron resecados. El post-operatorio fue satisfac. torio. En el estudio anátomo patológico de la pieza resecada habia evidencia de necrosis de ta pared. Al abrir el intestino habian tres pólipos necróticos, sésiles de más o menos $2 \mathrm{~cm}$. de diámetro constituidos, según el examen histológi$\infty$, por tejidos del tipo de los hamartomas. El tránsito de esófago, cstómago, duodeno, yeyuno e ileon realizado posteriormente reveló pólipos en el estómago $y$ en el yeyuno. La panendoscopía confirmo la poliposis gástrica. La enema baritada con técnica de doble contraste demustró un pólipo tipo Yamada II en el colon sigmoide. En la colonoscopía, que exploró hastá $90 \mathrm{~cm}$. del orificio anal externo, se observó un pólipo subpedunculado a $15 \mathrm{~cm}$. de distancia del orificio, que fue resecado en una colonoscopia efectuada posteriomnente. El estudio histológico del mismo demostró que también se trataba de un hamartoma. Su evolución fue satisfactoria.

J.M.A., niña de 9 años que fue admitida por dolor abdominal intenso, tipo cólico, vómitos y deterioro físico, de pocas horas de evolución. Tenía manchas melánicas en los labios, mucosa bucal, palma de las manos y planta de los pies y palidez de piel y mucosas. La palpación abdominal provocaba dolor, reacción de defensa muscular y permitía palpar una masa en el hemi- 
abdomen izquierdo. Ingresó con los diagnósticos de síndrome Peutz-Jeghers, obstrucción e inqa. ginación intestinales. En la intervención quirúrgica se encontró una gran maza de color negruzCo. que correspondía a una invaginación intestinal íleo-ileal, ubicada más o menos $90 \mathrm{~cm}$. antes de la válvula íleocecal, que no fue posible reducir, con evidente daño vascular. Se realizó resección de una zona de más o menos $20 \mathrm{~cm}$. de intestino, cuyo polo invaginante presentaba una masa polipoídea intraluminal y anastomosis término terminal. La paciente evolucionó bien $y$ fue dada de alta veinte días después del ingreso. En el examen anátomo patológico el trozo resecado correspondía a intestino delgado, tenía $20 \mathrm{~cm}$. de largo, mostraba marcada distensión y al abrirlo la porción invaginada presentaba exten. sa necrosis de la mucosa. En el examen histo. lógico, había necrosis hemorrágica extensa de la pared intestinal, donde la mucosa se proyectaba en el lumen, concluyéndose que se trataba de una lesión secundaria a invaginación intestinal, debida a síndrome de Peutz-Jeghers. En los controles posteriores se realizó tránsito intestinal que reveló presencia de pólipos en el estómago, el duodeno y la parte alta del yeyuno. La enema baritada mostró una imagen de pólipo, en el colon descendente. Mediante panendoscopía se vieron 3 pólipos grandes, pediculados, en la segunda y tercera porción del duodeno. En el estómago, cara anterior, próxima a la curvatura mayor, existía otro póljpo grande, de pedícuto largo y aspecto multilobulado. Durante la colonoscopía se observó un pólipo pediculado grande, a $60 \mathrm{~cm}$. del orificio anal externo, además de otros 5 pequeños pólipos sésiles. Se practicó polipectomía endoscópica del pólipo de mayor tamaño, cuyo estudio histológico demostró que correspondía a un hamartoma.

P.V.A., niño de Il años de edad, enviado al Policlinico de Gastroenterologia Infantil por presentar manchas melánicas en los labios, mucosa de la boca y pulpejos de los dedos. No tenía antecedentes de rectorragia ni dolor abdominal. La rectoscopía hasta $20 \mathrm{~cm}$. del orificio anal extemo mostró que habian pólipos pediculados a 13 y $5 \mathrm{~cm}$. de distancia y la enema baritada demostró pólipos en el colon. En las radiografías de tránsito de esófago, estómago y duodeno se encontró evidencia de dos lesiones solevantadas en el antro gástrico y en las de tránsito intestinal se encontraron algunos pólipos aislados en el yeyuno y. el íleon. Se introdujo un colonoscopio hasta $50 \mathrm{~cm}$. del orificio anal externo y se extirparon, mediante asa de alambre, los 2 pólipos rectales observados en la rectoscopía. $\mathrm{La}$

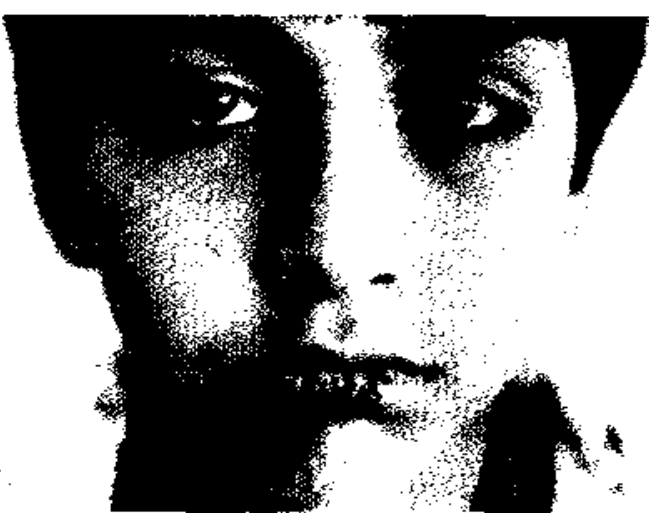

Figura 1: Manchas melánicas de la mucosa bucal características del Síndrome de Peutz-Jeghers.

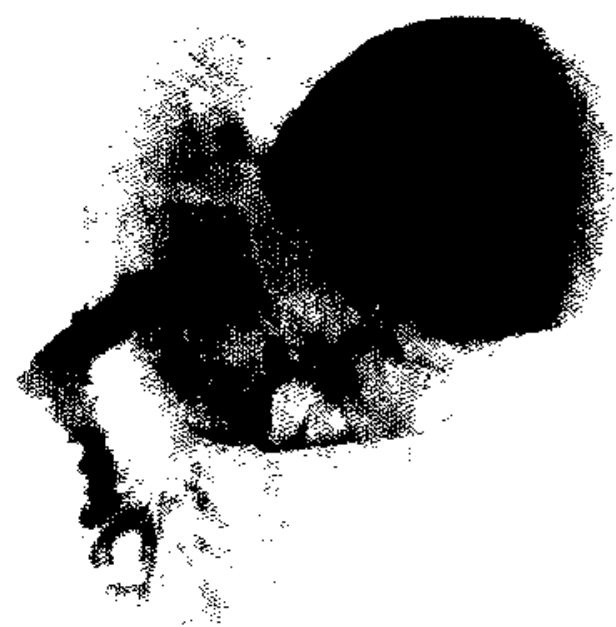

Figura 2: La Radiografia Esófago, Estómago y Duodeno demuestra en la curvatura mayor del estómago un pólipo grande y en el Duodeno varias masas polipoídeas.

esófago gastroduodenoscopía demostró un pequeño sobrelevantamiento de $3,5 \mathrm{~mm}$, en la curvatura mayor de la región angular que se extirpó mediante una biopsia térmica. Las caracteristicas histopatológicas de estos pólipos correspondían a las del síndrome de Peutz-Jeghers. Actualmente el paciente está en buenas condiciones $y$ : es controlado regularmente en el Policlínico de Gastroenterología.

\section{COMENTARIO}

Los sintomas más frecuentes en los pacientes con síndrome de Peutz-Jeghers son los cólicos 
intestinales, atribuibles a invaginaciones intestinales transitor las. Pueden asociarse con vómitos y sangramiento digestivo por ulceración e infartos de Jos pólipos y a obstrucción intestinal ${ }^{3}$.

Las invaginaciones colo-colónicas son las más frecuentes en los niños pequeños ${ }^{2}$. Las yeyuno ileales $\dot{y}$ las fleo ileales son más frecuentes en niños mayores y adultos y pueden constituir la primera manifestación de esta enfermedad 4,5

En esta serie, dos casos se manifestaron en la forma de invaginación del intestiro delgado y el tercero se pesquisó porque tenía manchas melánicas mucocutáneas. Los estudios radiológicos y endoscópicos permitieron detectar los múltiples pólipos del tracto gastrointestinal de estos pacientes.

La colonoscopia con fibra óptica es un méto. do efectivo para estudiar el colon y hacer el diagnóstico correcto, sobre todo porque permite estudiar segmentos más allá del alcance del rectoscopio. Además ofrece la posibilidad de tratamiento inmediato $y$ definitivo mediante polipectomía endoscópica ${ }^{6}$. Este tratamiento es preventivo si se realiza antes que ocurran complicaciones como en el tercer paciente.

El tratamiento quirúrgico se reserva para las complicaciones como la invaginación intestinal y sangramiento infestinal, o cuando el pólipo no se encuentra al alcance de la endoscopia.

La transformación maligna de los hamartomas deJ síndrome de Peutz--Jeghers ocurre en $2 \%$ de los $\operatorname{casos}^{a}$, siendo el duodeno el șegmento intest $\mathrm{i}$ nal donde esta complicación ocurte con mayor frecuencia. Por esta razón no se recomiendan actualmente las resecciones intestinales profilácticas $^{2}$.

\section{RESUMEN}

Se presentan tres pacientes con sindrome de Peutz- Jeghers. En dos de ellos su primera manifestación clínica fue la invaginación del intestino delgado. En el tercer paciente el diagnóstico se hizo por la presencia de las lesiones mucocutáneas típicas. En todos ellos se demostró la presencia de los pólipos en el tránsito de estómago e intestino delgado o en el enema baritado o la endoscopia alta a la colonoscopía. A gunos pólipos fueron extirpados durante este último procedimiento. Se destaca la utilidad de la polipectomía endoscópica.

\section{REFERENCIAS}

1. Jeghers H.H., Mc Kusick J.A.: Goneralized intestinal polyposis and melanin spots of the oral mucosa, lips and digits. $\lambda$. ling. J. Med. 241: 993.1949.

2. Howell $J$, Kevin $P_{\text {.: }}$ Peutz Jughers polyposis causing colocolic Intussusseption in Infancy. J. Ped. Surg. 16: 82, 1981 .

3. Silvernan A., Roy C.: Texbook Pediatric Clinical Gastroenterology chapter 11, pág. 308, St. Louis. The C. Mosby Co, 1983.

4. Monens P.M. Garvey S.P.: An unusual case of Peuts Jeghers Syndrome in an infant. Am. J. Dis. Child. 129: 173,1975 .

5. Wenel J.E., Bartholomew I.G.: Gastrointestinal polyposis with mucocutaneous pigmentation in children. Pcdiatrics 28: 655, 1961

6. Douglas J.R., Campbell C.A.: Colonoscopic polypectorny in children. Br. Mdd. J. 281: 1386, 1980.

7. Mckittnick, J.E., Lewis W.M.: The Pcutz Jeghers Syndrome. Arch Surg. 103: 57, 1971.

8. Dozois R.R. Itudd E.S.: The Peutz Jeghers Syndrome. Is there a prediposition to the development of intestinal malignancy? Arch Surg. 98: 509. 1969. 